\title{
Identification of EIL and ERF Genes Related to Fruit Ripening in Peach
}

\author{
Hui Zhou 1,2 , Lei Zhao 1,3, Qiurui Yang ${ }^{1,3}$, Mohamed Hamdy Amar ${ }^{1}{ }^{\oplus}$, Collins Ogutu ${ }^{1,4}$, \\ Qian Peng 1,3, Liao Liao ${ }^{1,4}$, Jinyun Zhang ${ }^{2}$ and Yuepeng Han 1,4,5,* \\ 1 CAS Key Laboratory of Plant Germplasm Enhancement and Specialty Agriculture, Wuhan Botanical Garden, \\ The Innovative Academy of Seed Design, Chinese Academy of Sciences, Wuhan 430074, China; \\ zhouhui@aaas.org.cn (H.Z.); zhaolei16@mails.ucas.ac.cn (L.Z.); yangqiurui16@mails.ucas.ac.cn (Q.Y.); \\ mohamed.amar@wbgcas.cn (M.H.A.); collins@wbgcas.cn (C.O.); pengqian114@mails.ucas.ac.cn (Q.P.); \\ liao168000@wbgcas.cn (L.L.) \\ 2 Key Laboratory of Genetic Improvement and Ecophysiology of Horticultural Crops, Institute of Horticulture, \\ Anhui Academy of Agricultural Sciences, Hefei 230031, China; zjy600@aaas.org.cn \\ 3 University of Chinese Academy of Sciences, 19A Yuquanlu, Beijing 100049, China \\ 4 Sino-African Joint Research Center, Chinese Academy of Sciences, Wuhan 430074, China \\ 5 Center of Economic Botany, Core Botanical Gardens, Chinese Academy of Sciences, Wuhan 430074, China \\ * Correspondence: yphan@wbgcas.cn; Tel./Fax: +86-27-8751-0872
}

Received: 25 March 2020; Accepted: 16 April 2020; Published: 19 April 2020

check for updates

\begin{abstract}
Peach (Prunus persica) is a climacteric fruit with a relatively short shelf life due to its fast ripening or softening process. Here, we report the association of gene families encoding ethylene insensitive-3 like (EIL) and ethylene response factor (ERF) with fruit ripening in peach. In total, 3 PpEILs and 12 PpERFs were highly expressed in fruit, with the majority showing a peak of expression at different stages. All three EILs could activate ethylene biosynthesis genes PpACS1 and PpACO1. One out of the 12 PpERFs, termed PpERF.E2, is a homolog of ripening-associated ERFs in tomato, with a consistently high expression throughout fruit development and an ability to activate PpACS1 and PpACO1. Additionally, four subgroup F PpERFs harboring the EAR repressive motif were able to repress the PpACO1 promoter but could also activate the PpACS1 promoter. Promoter deletion assay revealed that PpEILs and PpERFs could participate in transcriptional regulation of PpACS1 through either direct or indirect interaction with various cis-elements. Taken together, these results suggested that all three PpEILs and PpERF.E2 are candidates involved in ethylene biosynthesis, and EAR motif-containing $P p E R F s$ may function as activator or repressor of ethylene biosynthesis genes in peach. Our study provides an insight into the roles of EILs and ERFs in the fruit ripening process.
\end{abstract}

Keywords: Prunus persica; ethylene; ERF; EIL; fruit ripening

\section{Introduction}

Fruit ripening is a coordinated developmental process that leads to dramatic changes in the texture, color, flavor, and aroma of the fruit flesh. Ethylene plays a pivotal regulatory role in the fruit ripening process of most fleshy fruits [1-3]. Based on the intervention of ethylene during ripening, fruits can be divided into two categories: Climacteric fruits with a rapid rise in respiration and a burst of ethylene biosynthesis at the onset of ripening and non-climacteric fruits with no dramatic increase in respiration and ethylene biosynthesis. In climacteric fruits, the basal rate of ethylene production during development is often defined as system 1 ethylene, whilst system 2 refers to the high rate of autocatalytic ethylene synthesis during ripening [4].

The ethylene biosynthesis in plants starts with the precursor $S$-adenosylmethionine (S-AdoMet), which is synthesized from the methionine by $S$-AdoMet synthetase [5]. The $S$-AdoMet is converted to 
1-aminocyclopropane-1-carboxylic acid (ACC) by ACC synthase (ACS), and subsequently oxidized to ethylene via ACC oxidase (ACO). Both ACS and ACO are rate-limiting enzymes and encoded by multigene families [6-9]. Transcription factors (TFs) encoding ethylene-insensitive 3 (EIN3) and EIN3-like (EIL) proteins play important roles in the ethylene signaling of higher plants [5,7]. The EIN3 gene activates transcription of ethylene response genes, such as ERF1, through binding directly to a primary ethylene response element (PERE) $[10,11]$, whereas, the EIL gene can bind to various PERE-like elements [12,13]. The transcriptional activity of EILs is regulated by phosphorylation within the EIN3/EIL phosphorylation region (EPR), which promotes the dimerization process to initiate the EIL-mediated transcription of early ethylene-regulated genes [14,15]. The EIL genes show an expression divergence among species, with a peak level during fruit development [16-18] or ripening [19-21]. In addition, EILs may promote ethylene production by activating transcription of genes encoding rate-limiting enzymes in ethylene biosynthesis $[7,18]$.

ERF TFs belong to the APETALA2 (AP2)/ERF superfamily, which is characterized by a conserved AP2/ERF DNA binding domain, comprising of 60-70 amino acid residues [22]. There are 122 and 77 ERF TFs in the Arabidopsis and tomato genomes, respectively [22,23]. ERF TFs are involved in various biological processes, such as development [24,25], metabolism [26-28], plant hormone signaling [29-32], stress resistance [33-35], and fruit ripening [27,36,37]. ERF TFs regulate fruit ripening by directly binding to the GCC-box with a core AGCCGCC sequence or dehydration-responsive element (DRE) motif of their downstream genes, such as ACS and ACO3 of tomato [38,39]. However, both the GCC-box and DRE motif are absent in the promoter of ERF target genes, such as ACO54 in pear [40] and XET5 in kiwifruit [18], which suggests the possibility of other unknown cis-element(s) recognized by ERF TFs.

ERF TFs are divided into positive and negative regulators based on the activation or repression function of conserved motifs outside of the AP2/ERF domain [22]. Transcriptional activation motifs are probably related to acidic regions but show no sequence conservation [41]. By contrast, the transcriptional repression function is usually usually due to the ERF-associated amphiphilic repression (EAR) motif (LxLxL or DLNxxP) in the C-terminal region [18,42,43]. EAR motif-mediated repression probably occurs through chromatin modification of regulatory regions by histone deacetylation via physically interaction with co-repressors, such as Sin3-associated polypeptide of $18 \mathrm{kDa}$ (SAP18) or TOPLESS (TPL) [44,45].

Peach (Prunus persica) is a typical climacteric fruit with a short shelf life. PpACS1 (Prupe.2G176900) and PpACO1 (Prupe.3G209900) are the rate-limiting enzymes for autocatalytic ethylene synthesis during the ripening stage in peach [46-48]. In addition, isolation of EIL and ERF genes and their expression profiles have been reported in peach but with no functional assessment [49-51]. In this study, functional analysis was conducted for 3 members of the EIL family and 12 members of the ERF family using the dual luciferase assay. All three EIL genes, PpEIL1-3, and an ERF gene PpERF.E2 were found to be candidates involved in fruit ripening. Interestingly, the repressor-type $E R F$ genes showed the ability to activate transcription of the ethylene biosynthetic gene PpACS1. Our results provide an insight into ethylene signal transduction in the fruits of peach.

\section{Results}

\subsection{Identification of EIL and ERF Genes That Are Expressed in Peach Fruit}

Five putative EILs and 102 putative ERFs were recently reported in the peach genome [52]. Based on our previously reported database of the peach transcriptome [53], the expression levels of these $E I L$ and ERF genes were estimated using the FPKM value (fragments per kilobase of transcript per million mapped reads) in different tissues, including green- and red-colored young leaves, white and red flowers, and yellow- and red-fleshed fruits. Among the five EILs, four members, PpEIL1, PpEIL2, PpEIL3, and PpEIL5, were highly expressed in fruit, with the FPKM value greater than 20.0, while one member PpEIL4 showed no expression in all tested tissues (Table 1). PpEIL1, PpEIL2, and PpEIL3 encode putative proteins of 601, 601, and 624 amino acids in length, respectively. By contrast, PpEIL5 
encodes a putative protein with only 101 amino acids in length due to a truncated EIN3 domain, suggesting the possibility of PpEIL5 being a pseudogene.

Table 1. The Fragments Per Kilobase per Million (FPKM) value for the PpEIL genes in the leaf, flower, and fruit.

\begin{tabular}{ccccccccccc}
\hline \multirow{2}{*}{ Gene } & \multirow{2}{*}{ Accession No. } & \multicolumn{2}{c}{ Flower } & \multicolumn{2}{c}{ Leaf } & \multicolumn{4}{c}{ Fruit } \\
\cline { 3 - 10 } & & WF & RF & GL & RL & YS3 & RS3 & YS4 & RS4 \\
\hline PpEIL1 & Prupe.6G018200 & 13.2 & 13.0 & 13.5 & 15.6 & 12.5 & 15.6 & 15.3 & 21.6 \\
PpEIL2 & Prupe.2G058400 & 78.8 & 77.7 & 130.8 & 146.3 & 198.6 & 171.7 & 167.9 & 225.2 \\
PpEIL3 & Prupe.2G058500 & 42.9 & 35.8 & 68.9 & 68.9 & 61.5 & 44.0 & 46.8 & 65.5 \\
PpEIL4 & Prupe.6G181600 & 0.0 & 0.0 & 0.0 & 0.0 & 0.0 & 0.0 & 0.0 & 0.0 \\
PpEIL5 & Prupe.2G070300 & 14.8 & 13.4 & 19.2 & 23.1 & 22.2 & 22.6 & 8.1 & 30.6 \\
\hline
\end{tabular}

WF, white flower; RF, red flower; GL, green leaf; RL, red leaf; YS3, yellow-fleshed fruit at S3; RS3, red-fleshed fruit at S3; YS4, yellow-fleshed fruit at S4; RS4, red-fleshed fruit at S4. S3 and S4 represent the second exponential growth phase and ripening stage, respectively.

Out of the 102 PpERFs, 64 were expressed in at least one tissue, while 38 had no expression in all tested tissues. Subsequently, a heatmap was constructed to estimate the expression profile of these 64 expressed PpERFs based on the FPKM values (Figure 1). As a result, 12 PpERFs were found to be highly expressed in fruit, with FPKM > 10 in both yellow- and red-fleshed fruits at the ripening stage (S4), thus these were selected for further study.

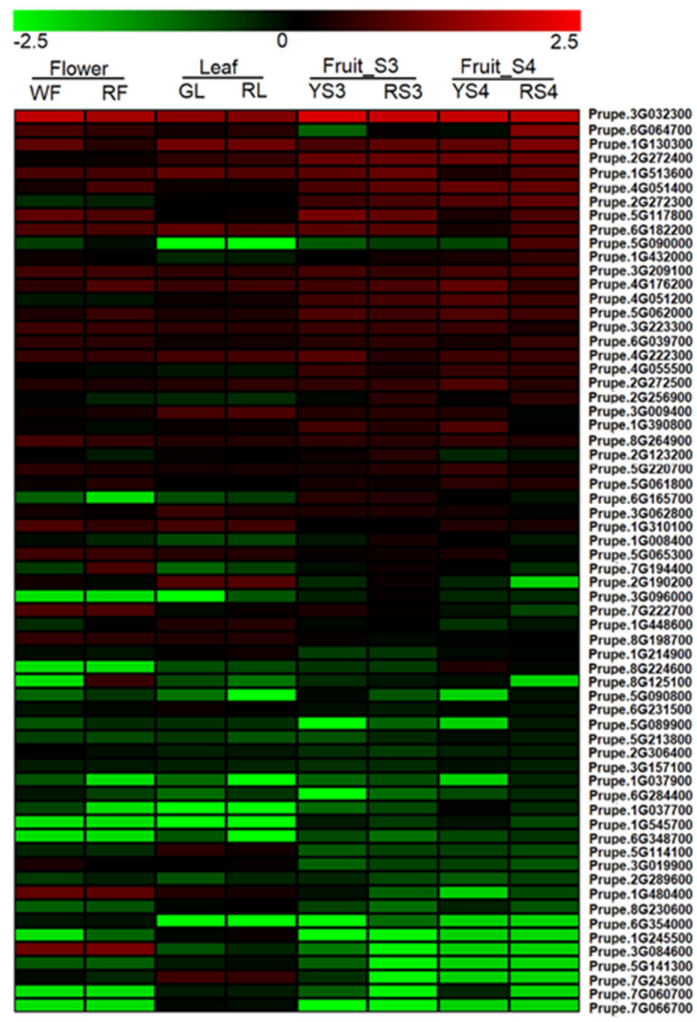

Figure 1. Heatmap of ethylene response factor (ERF) genes in the transcriptomes of different peach tissues. Red and green boxes indicate high- and low-expression levels, respectively. WF, white flower; RF, red flower; GL, green leaf; RL, red leaf; YS3, yellow-fleshed fruit at S3; RS3, red-fleshed fruit at S3; YS4, yellow-fleshed fruit at S4; RS4, red-fleshed fruit at S4. S3 and S4 represent the second exponential growth phase and ripening stage, respectively. 
Phylogenetic analysis revealed that the 12 PpERFs were classified into four subgroups, A, B, E, and $\mathrm{F}$ (Figure 2).

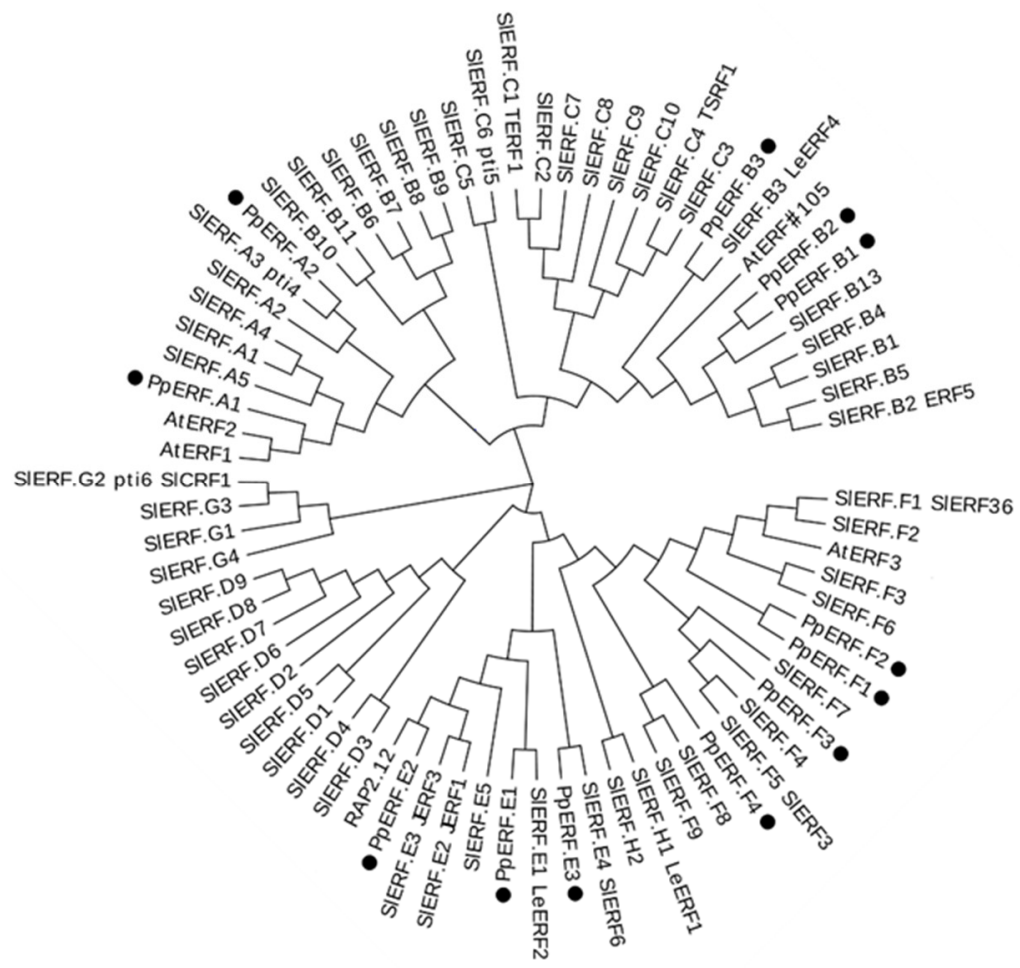

Figure 2. Phylogenetic tree derived from amino acid sequences of ERFs in peach, tomato, and Arabidopsis. The peach ERFs are highlighted with solid black circles. Full amino acid sequences of ERFs were aligned using Muscle software with default parameters, and a phylogenetic tree was constructed with MEGA software (version X) using the maximum likelihood method. All positions with less than $90 \%$ site coverage were eliminated, and ambiguous bases were allowed at any position (partial deletion option). The bootstrap consensus tree was inferred from 1000 replicates. All the tomato and Arabidopsis protein sequences were retrieved from Sol Genomics Network and Tair, respectively.

According to the nomenclature for the $E R F$ family in tomato, these 12 PpERFs were designated as follows: PpERF.A1 (Prupe.5G061800) and PpERF.A2 (Prupe.2G272300) in subgroup A; PpERF.B1 (Prupe.2G272500), PpERF.B2 (Prupe.2G272400), and PpERF.B3 (Prupe.5G062000) in subgroup B; PpERF.E1 (Prupe.8G264900), PpERF.E2 (Prupe.3G032300), and PpERF.E3 (Prupe.1G130300) in subgroup E; and PpERF.F1 (Prupe.3G209100), PpERF.F2 (Prupe.4G222300), PpERF.F3 (Prupe.4G051400), and PpERF.F4 (Prupe.4G051200) in subgroup F. The deduced amino acid sequences of the 12 PpERFs all contained a conserved AP2/ERF domain with variable location sites (Figure 3A). The AP2/ERF domain consisted of three $\beta$-sheets and one $\alpha$-helix (Figure 3B). In addition to the AP2/ERF domain, the EAR motif (DLNxxP), a repression domain, was also identified in subgroup F PpERFs, PpERF.F1, to PpERF.F4 (Figure $3 A, C$ ). This suggested that the subgroup F P $E R F$ s could have a negative regulatory activity. 


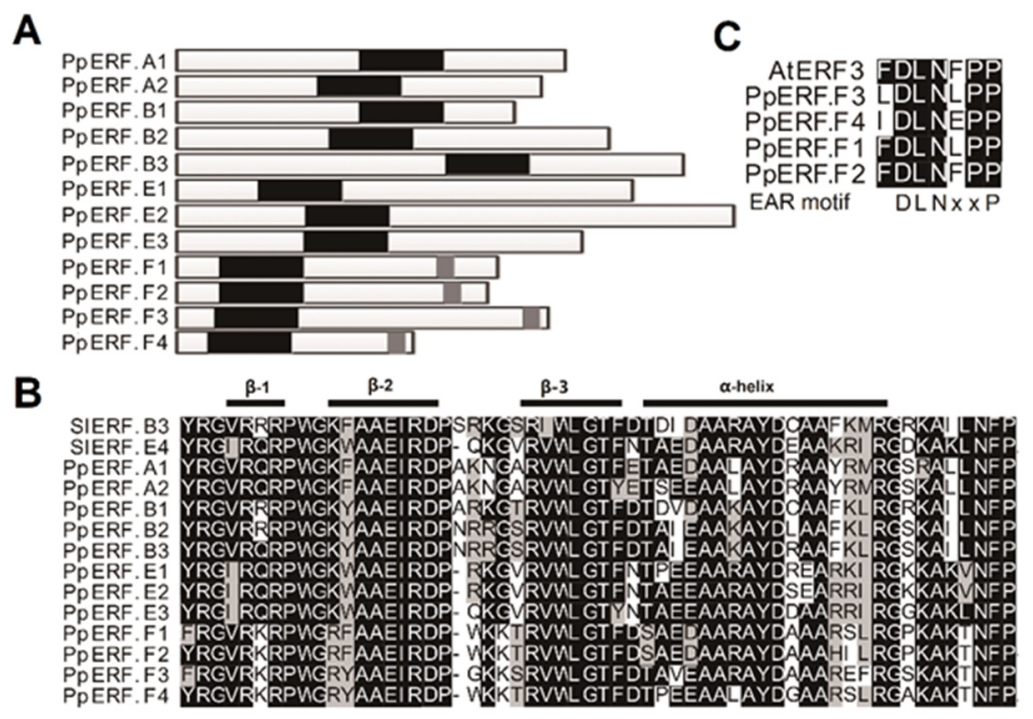

Figure 3. Diagram and alignment of ERF transcription factors (TFs). (A) structural diagram of the 12 PpERFs highly expressed in peach mesocarp. Black background boxes represent the AP2/ERF domain. The grey boxes stand for the EAR-repressing motif. $(\mathbf{B}, \mathbf{C})$ alignments of the EAR motif and AP2/ERF domain of the ERFs in peach and other species, respectively.

\subsection{Expression Profile of EIL and ERF Genes in Peach Fruit}

qRT-PCR was conducted to investigate the expression profile of EIL and ERF genes in fruits of cv. 'Zhong You 4' and its early ripening bud sport 'Li Xia Hong' at different developmental stages, including S1 (fruitlet), S2 (the first exponential growth phase), S3 (the second exponential growth stage), and S4 (the ripening stage). For the PpEIL genes, PpEIL1 had extremely lower levels of expression compared with PpEIL2 and PpEIL3. Both PpEIL2 and PpEIL3 were constitutively expressed during fruit development and ripening, with PpEIL3 showing consistently lower levels of expression in 'Zhong You 4' than in 'Li Xia Hong' (Figure 4).
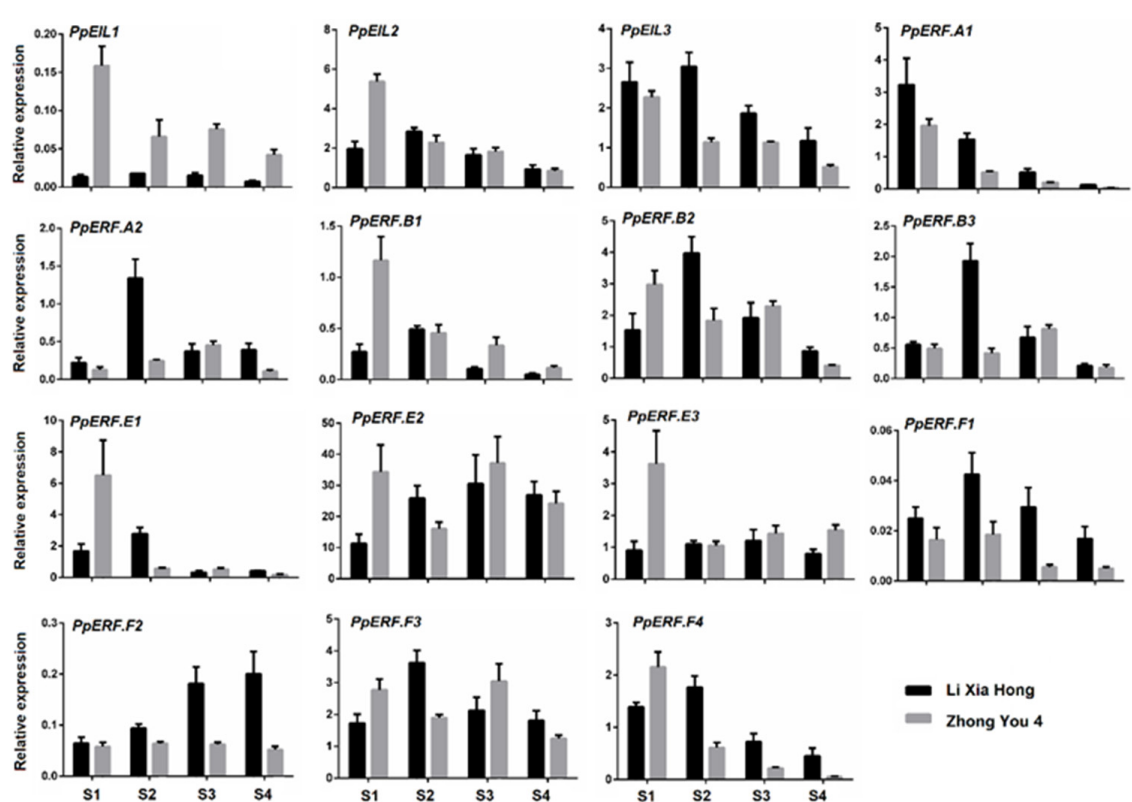

- Li Xia Hong
Zhong You 4

Figure 4. Expression profiles of the PpEILs and PpERFs in fruits of 'Zhong You 4' and its early ripening bud sport 'Li Xia Hong' at different developmental stages. The error bars show standard error (SE) of three biological replicates. 
A wide diversity of expression profiles were observed for the 12 PpERFS (Figure 4). Several PpERFs showed a decreasing trend in expression during fruit ripening. For example, the transcript abundance of three PpERFs, PpERF.A1, PpERF.B1, and PpERF.E1, was extremely low at the S4 stage of both 'Zhong You 4 ' and 'Li Xia Hong'. By contrary, PpERF.F2 showed an increasing trend in expression throughout the development of fruits in 'Li Xia Hong', with a peak at the fruit ripening stage. In addition, the expression levels of $P p E R F$.E2 remained consistently high throughout fruit development and were notably higher than those of other PpERFs.

\subsection{Roles of PpEILs and PpERFs in Transcriptional Regulation of Ethylene Biosynthesis Genes}

During the ripening process of fruits, EILs and ERFs participate in the fruit ripening process by regulating the expression of ethylene biosynthetic genes, $A C S$ and $A C O[7,18,26]$. In peach, PpACS1 and $P p A C O 1$ have been proven to be the rate-limiting genes for system 2 ethylene biosynthesis [46,54]. To clarify the role of PpEILS or PpERFs in transcriptional regulation of PpACS1 and PpACO1, cis-regulatory elements were screened in their promoter regions approximately 2 -kb upstream of the start codon using the PLACE program (Figure 5A). Two putative PERE-like motifs located 453 and $1291 \mathrm{bp}$ upstream of the start codon were identified for PpACS1, while only one putative PERE-like motif 1347 bp upstream of the start codon was found for PpACO1. Similarly, only one DRE box was found in the promoter of PpACS1, while one DRE and one GCC box were detected in the promoter of $P p A C O 1$. These results suggested potential roles of PpEILs and PpERFs in transcriptional regulation of PpACS1 and PpACO1.

To validate whether $P p E I L s$ and $P p E R F s$ had a direct role in the transcriptional activation of $P p A C S 1$ and PpACO1 promoters, a dual luciferase assay was carried out. For the PpACS1 promoter, Luciferase (Luc)/Renilla (Ren) values of PpEIL1, PpEIL2, and PpEIL3 were 5.2-, 6.0-, and 6.7-fold higher, respectively, than that of the empty vector control (Figure 5B). For the $P p A C O 1$ promoter, the Luc/Ren values of PpEIL1, PpEIL2, and PpEIL3 were 2.6-, 2.5-, and 2.9-fold higher, respectively, than that of the empty vector control. These results indicated that PpEIL1, PpEIL2, and PpEIL3 were able to activate the promoters of ethylene biosynthetic genes. Subsequently, the promoter activation activity was tested for eight activator-type P $p E R F s$ in subgroups A, B, and E (Figure 5C). Among the eight PpERF genes, PpERF.E2 showed the highest ability to activate the promoters of both PpACS1 and PpACO1, with a 2.6and 2.0-fold higher Luc/Ren value, respectively, than the empty vector control. Moreover, PpERF.E2 was phylogenetically related to tomato ripening-associated ERFs, such as SIERF.E1, SlERF.E2, and SIERF6/SIERF.E4 (Figure 2), with $47.7 \%, 58.1 \%$, and $37.8 \%$ amino acid sequence identities, respectively. Thus, PPERF.E2 was deemed a candidate involved in ethylene synthesis in peach.

Finally, the promoter repressive activity was tested for four repressor-type $P p E R F s$ in subgroup F, including PpERF.F1, PpERF.F2, PpERF.F3, and PpERF.F4, which all harbored the EAR motif (Figure 3A). Among the four repressor-type $P p E R F$ genes, $P p E R F . F 2$ showed the strongest repressive activity on the $P p A C O 1$ promoter, with an approximately 5-fold lower Luc/Ren value than the empty vector control (Figure 6A). Surprisingly, all the subgroup F PpERFs showed high levels of activation activity instead of repressive activity on the PpACS1 promoter. Among all the tested PpERFs, PpERF.F2 showed the strongest ability to activate the PpACS1 promoter, with a 30.0-fold higher Luc/Ren value than the empty vector control. Altogether, these results suggested that subgroup F ERFs in peach exhibit a potential dual function and could repress or activate gene transcription depending on the promoter in which they act. 


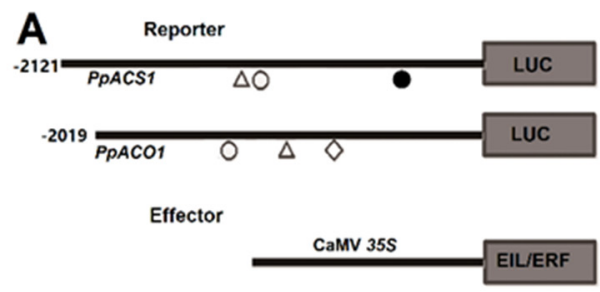

B
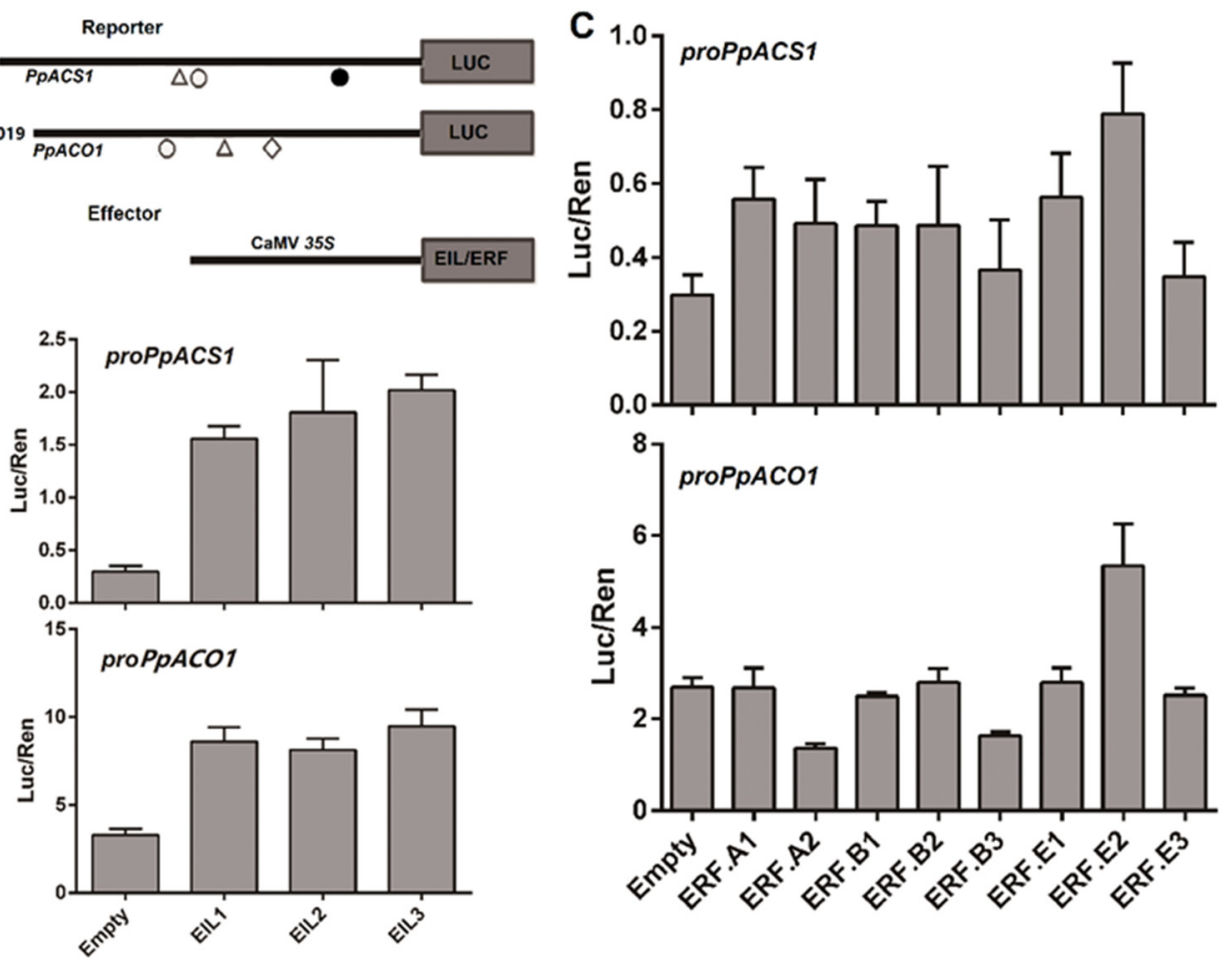

Figure 5. Functional analysis of the promoter sequences of PpACS1 and PpACO1. (A) Construction of the effector and reporter cassettes. Cis-elements were indicated by triangle (DRE motif), black circle (PERE motif), white circle (PERE-like motif), and diamond (GCC-box). (B,C) Analysis of the interaction of PpEILs and activator-type PpERFs with the promoters of PpACS1 and PpACO1 using the dual luciferase assay in young leaves of 3- to 4-week-old Nicotiana benthamiana seedlings. The error bars represent SE of four biological replicates.

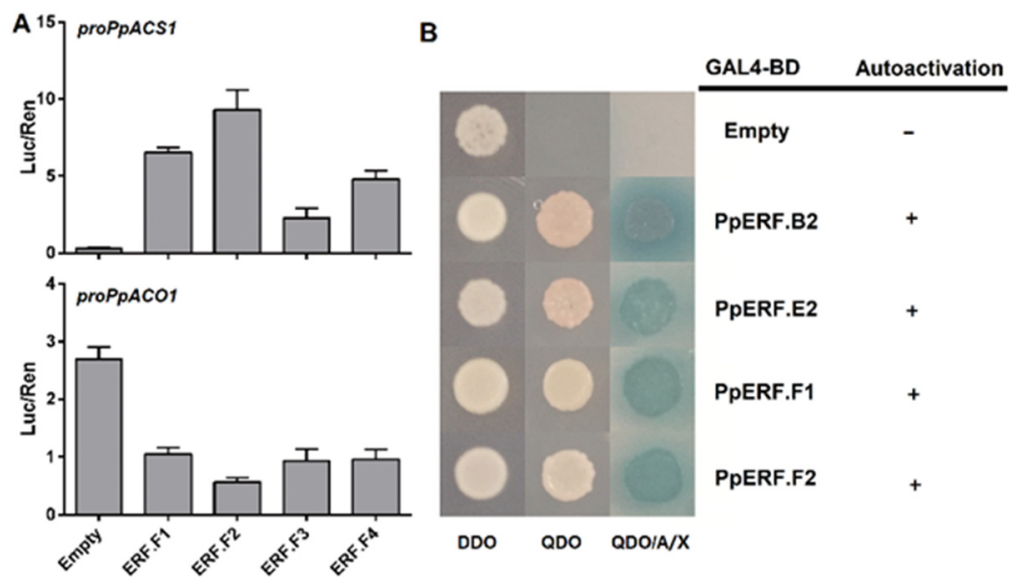

Figure 6. Functional analysis of group F ERFs. (A) Assay of the activation of group $F$ ERFs on the promoter of PpACS1 and PpACO1 using the dual luciferase assay. Empty vector was used as the control. The error bars show SE of four biological replicates. (B) Auto-activation activity test of PpERFs in yeast. The yeast was grown on three medium Minimal Media Double Dropouts (DDO), Minimal Media Quadruple Dropouts (QDO), and QDO with Aureobasidin A and X-a-Gal (QDO/A/X), corresponding to SD-Trp, SD-Trp-His-Ade, and SD-Trp-His-Ade+ABA+X- $\alpha-G a l$, respectively. 


\subsection{Analysis of Transcriptional Activation Ability of PpERF Proteins in Yeast}

The N- and C-terminal regions of ERF TFs contain transcriptional activation or repression domains [22]. To investigate whether subgroup F PpERFs also contain an activation domain, in addition to the EAR repression domain, full-length coding sequences of PpERF.F1 and PPERF.F2 were individually inserted into the PGBKT7 vector, and then transformed into yeast strain Y2Hgold to test their auto-activation activity in yeast cells. Activator-type ERFs, PpERF.B2 and PpERF.E2, were used as positive controls. Yeast cells carrying either positive control genes or subgroup F P $p E R F$ genes were able to grow on the SD-Trp-His-Ade+ABA medium, and their color turned into blue when grown on media containing $\mathrm{X}-\alpha$-Gal (Figure $6 \mathrm{~B}$ ). This result suggested the presence of an activation domain, besides the EAR repressive domain, in proteins encoded by he PpERF.F1 and PpERF.F2 genes, which endowed them with potential dual functions: Acting as both an activator and repressor in peach.

\subsection{Identification of Cis-Elements in the PpACS1 Promoter That Interact with EIL and ERF TFs}

To identify cis-elements interacting with PpEILs, PpERF.E2 and subgroup F ERFs, four different promoter constructs of $P p A C S 1$, ranging from 0.4 to $2.1 \mathrm{~Kb}$ upstream of the start codon, were selected to conduct the dual luciferase assay (Figure 7A). PpEIL3 showed an almost equal ability to activate either P0 or P1 promoters but with a dramatically reduced ability to activate both $\mathrm{P} 2$ and $\mathrm{P} 3$ deletion constructs (Figure 7B). This indicated that PpEIL3 interacted with the PERE-like motif (ATTCAAA). Similarly, PpERF.F2 showed the strongest ability to activate the P0 promoter but with a dramatically reduced ability to activate $\mathrm{P} 1$ and $\mathrm{P} 2$ deletion constructs that both contained a deletion of the DRE motif (GTCGG) as compared to the P0 promoter. Therefore, PpERF.F2 is likely to interact with the DRE motif. However, PpERF.E2 showed the similar ability to activate all four promoters, suggesting that PpERF.E2 probably activated the PpACS1 promoter in an indirect way. Taken together, these results suggested that PpEILs and PpERFs could regulate the transcription of PpACS1 through either direct or indirect interaction with various cis-elements in the promoter region.
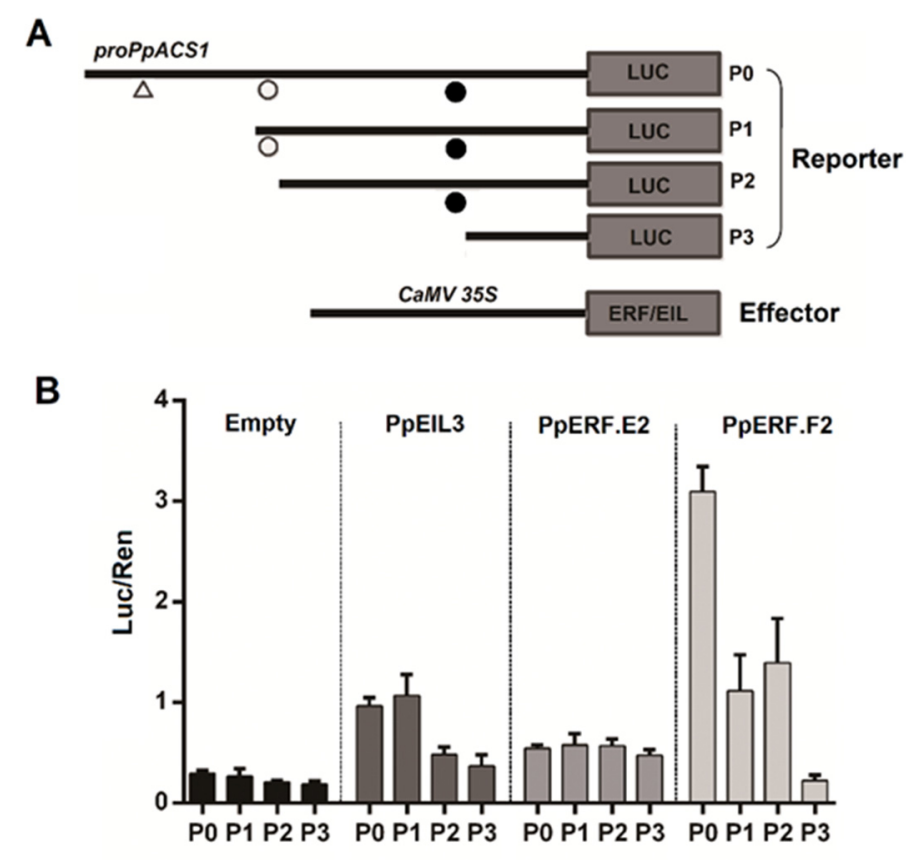

Figure 7. Promoter deletion analysis of PpACS1. (A) Four promoter deletion constructs used for the construction of reporter cassette and ERF/EIL genes related to the effector cassette. The predicted DRE, PERE-like, and PER motifs are indicated by a triangle, white circle, and black circle, respectively. (B) Assay of the activation effect of PpEIL3, PpERF.E2, and PpERF.F2 on four different length sequences of P ACS1 using the dual luciferase assay. The error bars show SE of four biological replicates. 


\section{Discussion}

The fruit ripening process in climacteric fruits is tightly associated with ethylene biosynthesis, which is mediated by the auto-inhibitory system 1 in unripe fruit and autocatalytic system 2 in ripening fruit $[6,7]$. Although a positive feedback loop between ethylene signaling and biosynthesis is known to control the autocatalytic system 2, its associated TFs and pathway are yet to be determined. A recent study showed that eudicots with or without recent whole-genome duplication (WGD) utilize the MADS- or NAC-type positive feedback loops, respectively, while monocots, such as banana, uses both MADS-box and NAC TFs to form a dual-loop system [55]. This suggests that MADS-box and NAC genes are involved in ethylene biosynthesis, acting as regulators that induce transcription of ethylene biosynthesis genes $A C S$ and $A C O$. An MADS-type positive feedback loop seems to be true in tomato as the MADS-box gene RIN is weakly expressed in young fruit but highly expressed in mature green and breaker stages [56]. In peach, $P p N A C 1$ is reported to be involved in an NAC-type postive feedback loop [55]. However, $P p N A C 1$ shows low levels of expression in early stages of fruit development, but with a high level of expression in the second exponential growth stage (S3) before the initial activation of ethylene biosynthesis [53]. Thus, it is more likely that other regulatory gene(s) instead of $P p N A C 1$ may play critical roles in ethylene biosynthesis despite the transcriptional regulation of $A C S$ and ACO by PPNAC1 [55].

Besides MADS-box and NAC genes, other TFs at the late step of ethylene signal transduction, such as EIN3/EIL and ERF, are also known to participate in ethylene synthesis $[17,18]$. EIN3/EIL proteins are post-translationally regulated in response to the ethylene signal in Arabidopsis [57]. Several EILs that are constitutively expressed throughout fruit development have been reported in climacteric fruits, such as tomato and kiwifruit, although their expression levels are relatively lower during the ripening stage than in the early stages of fruit development $[17,18]$. A similar phenomena was also observed for peach EILs in this study. All three PpEILs can activate the transcription of both PpACS1 and PpACO1, which is consistent with the previous finding in tomato and kiwifruit [7,18]. In Arabidopsis, ubiquitin or proteasome-dependent proteolysis mediates the post-translational regulation of EIN3 by two F-box proteins, EBF1 and EBF2 [58,59]. Given the fact that ethylene remains at a basal level in climacteric fruits at the juvenile stage, it is reasonable to speculate that the expression of EILs in climacteric fruits, such as peach, is also regulated at the post-translational level. Moreover, EIN3/EIL are known to activate ethylene response genes, such as AtERF1, OsEBP-89, and NtTERF1, through binding to the PERE or PERE-like cis-elements in their promoter regions $[10,12,13]$. Interestingly, PpEIL3 interacts with the PERE-like motif (ATTCAAA) found in the promoter of TERF1, which is regulated by EILs in tobacco, rather than the PERE motif (TTCAAAT) found in the promoter of Os-EBP89 encoding an ethylene responsive element binding protein in rice [13]. Thus, it seems that the divergence of EIL-binding motifs has occurred during the process of speciation in plants. In addition, all three EIL genes can activate the promoters of PpACS1 and PPACO1, suggesting a redundant role of the EIL gene family in ethylene biosynthesis.

The ERF family is divided into two subfamilies, CBF/DREB and ERF. Subgroups B and E of the ERF subfamily have been reported to participate in the fruit ripening process [22,60]. In tomato, a subgroup B member Sl-ERF.B3 and a subgroup E member LeERF2/TERF2 positively or negatively regulate the expression of both $A C S$ and $A C O$ genes, respectively [38,61], suggesting that subgroups $\mathrm{B}$ and $\mathrm{E}$ of the ERF subfamily may be involved in ethylene biosynthesis. Here, three subgroup $\mathrm{E}$ members, PpERF.E1 - P PERF.E3, were highly expressed in fruit, but only PpERF.E2 had ability to activate $P p A C S 1$ and $P p A C O 1$. This suggests that $P p E R F$.E2 participates in ethylene biosynthesis. By contrast, three subgroup B members, PpERF.B1 to PpERF.B3, which were highly expressed in fruit, were all unable to activate either PpACS1 or PpACO1 (Figure 5C). Previous studies show that ERF genes can form a hierarchical transcriptional cascade to regulate the ripening process in tomato [23] and apple [39]. Thus, it is worthy of further study to ascertain whether subgroup B members of the ERF subfamily can act as upstream regulators of PpERF.E2. 
The F subgroup ERF TFs harboring the EAR motif (LxLxL or DLNxxP) in the C-terminus function as repressors to negatively regulate photosynthesis, stress response, cell wall metabolism, and fruit ripening [18,26,61]. Overexpression of the F subgroup ERF TFs, such as ERF3 in cotton [35] and MaERF11 in banana [62], results in downregualtion of $A C O$ and ACS. Interestingly, our results showed that all the four F subgroup ERF TFs harboring the EAR motif, PpERF.F1—PpERF.F4, could play a dual function by activating PpACS1 and repressing PpACO1. ERF TFs usually regulate fruit ripening by directly binding to the GCC-box or DRE motif of their downstream genes [38,39], and previously reported EAR-motif-containing ERF TFs are found to bind to the GCC box but not the DRE motif [42,62]. Nevertheless, one DRE cis-element instead of the GCC box in the promoter region of PpACS1 is most likely interact with PpERF.F2 in this study, indicating the possibility that EAR-motif-containing ERF repressors directly activate P $P A C S 1$ in peach. However, it cannot be ruled out that the peach ERF repressors indirectly activate $P p A C S 1$ through interacting with other DRE-motif-containing regulators involved in fruit ripening.

\section{Methods}

\subsection{Plant Material}

Peach variety 'Zhong You 4' and its early ripening bud sport 'Li Xia Hong' are maintained in Institute of Horticulture, Anhui Academy of Agricultural Sciences, Hefei 230031, China. For 'Zhong You 4', fruit samples were collected at 15, 31, 47, and 63 days after flower bloom (DAFB), corresponding to S1 (fruitlet), S2 (the first exponential growth phase), S3 (the second exponential growth stage), and S4 (the ripening stage), respectively. For 'Li Xia Hong', fruit samples were also collected at 15 (S1), 31 (S2), 55 (S3), and 79 (S4) DAFB. Fruit samples were peeled, cored, and cut into small pieces; frozen immediately in liquid nitrogen; and then stored at $-75^{\circ} \mathrm{C}$ until use. Each cultivar at each stage had three biological replicates, with each containing at least five fruits.

\subsection{Heatmap Analysis of the ERF Gene Family}

RNA-seq data were retrieved and analyzed according to our previous report [53]. Gene expression levels in leaves, flowers, and fruits were quantified using FPKM values (fragments per kilo bases per million reads). The heatmap was created using MeV (Multiple Experiment Viewer) 4.90 software [63].

\subsection{RNA Extraction and Quantitative Real-Time PCR ( $q R T-P C R)$}

Approximately 100-mg fruit samples were ground into powder in liquid nitrogen and then subjected to total RNA extraction using the Total RNA Rapid Extraction Kit (Zomanbio, Beijing 100085, China) according to the manufacturer's instructions. RNA extracts were treated with DNase I and then converted to cDNA using the PrimeScript ${ }^{\mathrm{TM}}$ RT reagent Kit with gDNA Eraser (Takara, Dalian 116000, China). qRT-PCR was conducted using TB GREEN (Takara Bio, Inc.), following the manufacturer's instructions on an ABI StepOne Plus real-time PCR system (Applied Biosystems, Foster City, CA, USA). The amplification program was as follows: One cycle of $30 \mathrm{~s}$ at $95^{\circ} \mathrm{C}$, followed by 40 cycles of $5 \mathrm{~s}$ at $95{ }^{\circ} \mathrm{C}$ and $34 \mathrm{~s}$ at $60{ }^{\circ} \mathrm{C}$. Translation elongation factor 2 (TEF2) was used as a constitutive control according to the previous report [64]. All analyses were repeated three times using biological replicates. Primer sequences used for qRT-PCR are listed in Table S1.

\subsection{Dual Luciferase Reporter Assay}

The dual luciferase reporter assay was conducted as previous reports [65,66]. Briefly, the 2.1- and 2.0-kb regions upstream of the start codon in PpACS1 and PpACO1, respectively, were amplified and inserted into the reporter vector pGreenII 0800-LUC+, whereas the effector vectors were constructed by insertion of the full-length coding sequences of EIL and ERF genes into the binary expression vector pSAK277 under the CaMV $35 S$ promoter. Four PpACS1 promoter deletion constructs, P0, P1, P2, and 
P3, containing 2.12-, 1.31-, 1.28-, and 0.45-kb DNA fragments upstream of the start codon, respectively, were selected to conduct the dual luciferase assay.

Both the reporter and effector constructs were individually transformed into Agrobacterium strain GV3101 harboring the pSoup helper plasmid. After incubation at $28^{\circ} \mathrm{C}$ for 2 days, a $10-\mu \mathrm{L}$ loop of confluent bacteria was re-suspended in $10 \mathrm{~mL}$ of infiltration buffer containing $10 \mathrm{mM} \mathrm{MgCl}_{2}, 200 \mu \mathrm{M}$ acetosyringone, and $10 \mathrm{mM} 2-(\mathrm{N}$-morpholine)-ethanesulfonic acid $(\mathrm{pH}=5.7)$, and incubated at room temperature without shaking for $2 \mathrm{~h}$. Agrobacterium cultures containing the reporter cassette or the effector construct were mixed at a ratio of 1:9. The mixed cultures of bacteria were injected into young leaves of Nicotiana benthamiana seedlings by syringes without a needle. The seedlings were cultured in a greenhouse at $25^{\circ} \mathrm{C}$ with a $16 / 8 \mathrm{~h}$ light/darkness cycle for 3 days. Leaf disks $(2 \mathrm{~cm}$ in diameter) adjacent to the infiltration site were punched to measure the Luc and Ren luciferase activity using the Dual-Glo ${ }^{\circledR}$ Luciferase Assay System (Promega, Madison, WI 53711, USA) on an Infinite M200 luminometer (Tecan, Mannerdorf, Switzerland).

\subsection{Measurement of Transcriptional Activation Activity of ERFs in Yeast}

The whole coding regions of PpERF.B2, PpERF.E2, PpERF.F1, and PpERF.F2 were cloned, inserted into the pGBKT7 vector, and individually transformed into the yeast strain ' $Y 2 \mathrm{Hgold}$ ' according to the instruction of the Frozen-EZ Yeast Transformation II ${ }^{\mathrm{TM}}$ (ZYMO RESEARCH CORP, Irvine, CA 92614, USA). Positive clones were grown on the SD-Trp or SD-Trp-His-Ade+AbA medium to test the transcriptional activation activity. Photographs were taken 3 days after incubation on medium.

\section{Conclusions}

In total, 3 P $p E I L$ genes and 12 PpERF genes showing relatively high expression in fruit were investigated for their relationship with ethylene biosynthesis in peach. All three EILs were able to activate the promoters of ethylene biosynthesis genes PpACS1 and PpACO1, suggesting that they are potentially involved in ethylene biosynthesis. Of the 12 PpERFs, PpERF.E2 was deemed a candidate involved in ethylene biosynthesis, as it showed a high expression as well as an ability to activate the transcription of $P p A C S 1$ and PpACO1. Interestingly, four subgroup F PpERFs harboring the EAR repressive motif are not only able to repress the PpACO1 promoter but can also activate the PpACS1 promoter. Thus, $P p E R F s$ P $p E R F s$ could function as an activator or repressor of ethylene biosynthesis genes in peach. Our study provides an insight into the roles of EILS and ERFs in the fruit ripening of peach.

Supplementary Materials: The following are available online at http:/www.mdpi.com/1422-0067/21/8/2846/s1, Table S1. Sequences of primers used for cloning genes and vectors construction and qRT-PCR.

Author Contributions: Y.H. and H.Z. conceived and designed the experiments; H.Z., L.Z., Q.Y., and Q.P. performed the experiments; Y.H. and H.Z. wrote the paper; and M.H.A., C.O., L.L. and J.Z. revised the manuscript. All authors have read and approved the manuscript.

Funding: This project was supported by funds received from the Special Fund for Strategic Pilot Technology of Chinese Academy of Sciences (XDA24030404-4), the National Natural Science Foundation of China (31601740), the China Agriculture Research System (grant no. CARS-30), and Science and Technology Major Project of Anhui Province (17030701011).

Conflicts of Interest: The authors declare no conflict of interest

\section{References}

1. Giovannoni, J. Molecular biology of fruit maturation and ripening. Annu. Rev. Plant Biol. 2001, 52, 725-749. [CrossRef] [PubMed]

2. Giovannoni, J.J. Fruit ripening mutants yield insights into ripening control. Curr. Opin. Plant Biol. 2007, 10, 283-289. [CrossRef] [PubMed]

3. Seymour, G.B.; Østergaard, L.; Chapman, N.; Knapp, S.; Martin, C. Fruit Development and Ripening. Annu. Rev. Plant Biol. 2013, 64, 219-241. [CrossRef] [PubMed] 
4. McMurchie, E.J.; McGlasson, W.B.; Eaks, I.L. Treatment of fruit with propylene gives information about the biogenesis of ethylene. Nature 1972, 237, 235-236. [CrossRef]

5. Wang, K.L.-C.; Li, H.; Ecker, J.R. Ethylene Biosynthesis and Signaling Networks. Plant Cell 2002, 14, s131-s151. [CrossRef]

6. Barry, C. The Regulation of 1-Aminocyclopropane-1-Carboxylic Acid Synthase Gene Expression during the Transition from System-1 to System-2 Ethylene Synthesis in Tomato. Plant Physiol. 2000, 123, 979-986. [CrossRef]

7. Yokotani, N.; Nakano, R.; Imanishi, S.; Nagata, M.; Inaba, A.; Kubo, Y. Ripening-associated ethylene biosynthesis in tomato fruit is autocatalytically and developmentally regulated. J. Exp. Bot. 2009, 60, 3433-3442. [CrossRef]

8. Barry, C.; Blume, B.; Hamilton, A.J.; Bouzayen, M.; Cooper, W.; Grierson, D. Differential expression of the 1-aminocyclopropane-1-carboxylate oxidase gene family of tomato. Plant J. 1996, 9, 525-535. [CrossRef]

9. Nakatsuka, A.; Murachi, S.; Okunishi, H.; Shiomi, S.; Nakano, R.; Kubo, Y.; Inaba, A. Differential expression and internal feedback regulation of 1-aminocyclopropane-1-carboxylate synthase, 1-aminocyclopropane1-carboxylate oxidase, and ethylene receptor genes in tomato fruit during development and ripening. Plant Physiol. 1998, 118, 1295-1305. [CrossRef]

10. Chao, Q.; Rothenberg, M.; Solano, R.; Roman, G.; Terzaghi, W.; Ecker, J.R. Activation of the ethylene gas response pathway in Arabidopsis by the nuclear protein ETHYLENE-INSENSITIVE3 and related proteins. Cell 1997, 89, 1133-1144. [CrossRef]

11. Solano, R.; Stepanova, A.N.; Chao, Q.; Ecker, J.R. Nuclear events in ethylene signaling: A transcriptional cascade mediated by ETHYLENE-INSENSITIVE3 and ETHYLENE-RESPONSE-FACTOR1. Genome Res. 1998, 12, 3703-3714. [CrossRef] [PubMed]

12. Mao, C.; Wang, S.; Jia, Q.; Wu, P. OsEIL1, a Rice Homolog of the Arabidopsis EIN3 Regulates the Ethylene Response as a Positive Component. Plant Mol. Biol. 2006, 61, 141-152. [CrossRef] [PubMed]

13. Zhang, H.; Li, A.; Zhang, Z.; Huang, Z.; Lu, P.; Zhang, D.; Liu, X.; Zhang, Z.-F.; Huang, R. Ethylene Response Factor TERF1, Regulated by ETHYLENE-INSENSITIVE3-like Factors, Functions in Reactive Oxygen Species (ROS) Scavenging in Tobacco (Nicotiana tabacum L.). Sci. Rep. 2016, 6, 29948. [CrossRef] [PubMed]

14. Li, J.; Tang, L.; Yang, Y.; Zouine, M.; Bouzayen, M. A conserved phosphorylation site regulates the transcriptional function of ETHYLENE-INSENSITIVE3-like1 in tomato. J. Exp. Bot. 2011, 63, 427-439. [CrossRef]

15. Quan, R.; Wang, J.; Yang, D.; Zhang, H.; Zhang, Z.; Huang, R. EIN3 and SOS2 synergistically modulate plant salt tolerance. Sci. Rep. 2017, 7, 44637. [CrossRef]

16. Tieman, D.M.; Ciardi, J.A.; Taylor, M.G.; Klee, H.J. Members of the tomato LeEIL (EIN3-like) gene family are functionally redundant and regulate ethylene responses throughout plant development. Plant J. 2001, 26, 47-58. [CrossRef]

17. Yokotani, N.; Tamura, S.; Nakano, R.; Inaba, A.; Kubo, Y. Characterization of a novel tomato EIN3-like gene (LeEIL4). J. Exp. Bot. 2003, 54, 2775-2776. [CrossRef]

18. Yin, X.-R.; Allan, A.C.; Chen, K.-S.; Ferguson, I.B. Kiwifruit EIL and ERF Genes Involved in Regulating Fruit Ripening1[W]. Plant Physiol. 2010, 153, 1280-1292. [CrossRef]

19. Tacken, E.; Ireland, H.; Wang, Y.-Y.; Putterill, J.; Schaffer, R. Apple EIN3 BINDING F-box 1 inhibits the activity of three apple EIN3-like transcription factors. AoB Plants 2012, 2012, 034. [CrossRef]

20. Huang, S.; Sawaki, T.; Takahashi, A.; Mizuno, S.; Takezawa, K.; Matsumura, A.; Yokotsuka, M.; Hirasawa, Y.; Sonoda, M.; Nakagawa, H.; et al. Melon EIN3-like transcription factors (CmEIL1 and CmEIL2) are positive regulators of an ethylene- and ripening-induced 1-aminocyclopropane-1-carboxylic acid oxidase gene (CM-ACO1). Plant Sci. 2010, 178, 251-257. [CrossRef]

21. Cao, Y.; Han, Y.; Meng, D.; Li, D.; Jin, Q.; Lin, Y.; Cai, Y. Genome-wide analysis suggests high level of microsynteny and purifying selection affect the evolution of EIN3/EIL family in Rosaceae. PeerJ 2017, 5, e3400. [CrossRef] [PubMed]

22. Nakano, T.; Suzuki, K.; Fujimura, T.; Shinshi, H. Genome-Wide Analysis of the ERF Gene Family in Arabidopsis and Rice. Plant Physiol. 2006, 140, 411-432. [CrossRef] [PubMed] 
23. Liu, M.; Gomes, B.L.; Mila, I.; Purgatto, E.; Peres, L.E.; Frasse, P.; Maza, E.; Zouine, M.; Roustan, J.-P.; Bouzayen, M.; et al. Comprehensive Profiling of Ethylene Response Factor Expression Identifies Ripening-Associated ERF Genes and Their Link to Key Regulators of Fruit Ripening in Tomato. Plant Physiol. 2016, 170, 1732-1744. [CrossRef] [PubMed]

24. Yin, C.-C.; Ma, B.; Collinge, D.P.; Pogson, B.J.; He, S.-J.; Xiong, Q.; Duan, K.-X.; Chen, H.; Yang, C.; $\mathrm{Lu}, \mathrm{X}$; et al. Ethylene responses in rice roots and coleoptiles are differentially regulated by a carotenoid isomerase-mediated abscisic acid pathway. Plant Cell 2015, 27, 1061-1081. [CrossRef] [PubMed]

25. Harkey, A.; Watkins, J.; Olex, A.L.; DiNapoli, K.; Lewis, D.R.; Fetrow, J.; Binder, B.; Muday, G.K. Identification of Transcriptional and Receptor Networks That Control Root Responses to Ethylene. Plant Physiol. 2017, 176, 2095-2118. [CrossRef] [PubMed]

26. Lee, J.M.; Joung, J.-G.; McQuinn, R.P.; Chung, M.-Y.; Fei, Z.; Tieman, D.; Klee, H.; Giovannoni, J.J. Combined transcriptome, genetic diversity and metabolite profiling in tomato fruit reveals that the ethylene response factor SIERF6 plays an important role in ripening and carotenoid accumulation. Plant J. 2012, 70, 191-204. [CrossRef]

27. Yin, X.-R.; Xie, X.; Xia, X.; Yu, J.; Ferguson, I.B.; Giovannoni, J.J.; Chen, K.-S. Involvement of an ethylene response factor in chlorophyll degradation during citrus fruit degreening. Plant J. 2016, 86, 403-412. [CrossRef]

28. Li, X.; Xu, Y.; Shen, S.; Yin, X.; Klee, H.; Zhang, B.; Chen, K. Transcription factor CitERF71 activates the terpene synthase gene CitTPS16 involved in the synthesis of E-geraniol in sweet orange fruit. J. Exp. Bot. 2017, 68, 4929-4938. [CrossRef]

29. Robles, L.; Stepanova, A.N.; Alonso, J. Molecular Mechanisms of Ethylene-Auxin Interaction. Mol. Plant 2013, 6, 1734-1737. [CrossRef]

30. Tadiello, A.; Longhi, S.; Moretto, M.; Ferrarini, A.; Tononi, P.; Farneti, B.; Busatto, N.; Vrhovsek, U.; Molin, A.D.; Avanzato, C.; et al. Interference with ethylene perception at receptor level sheds light on auxin and transcriptional circuits associated with the climacteric ripening of apple fruit (Malus $\mathrm{x}$ domestica Borkh.). Plant J. 2016, 88, 963-975. [CrossRef]

31. Thagun, C.; Imanishi, S.; Kudo, T.; Nakabayashi, R.; Ohyama, K.; Mori, T.; Kawamoto, K.; Nakamura, Y.; Katayama, M.; Nonaka, S.; et al. Jasmonate-responsive erf transcription factors regulate steroidal glycoalkaloid biosynthesis in tomato. Plant Cell Physiol. 2016, 57, 961-975. [CrossRef] [PubMed]

32. Bakshi, A.; Piya, S.; Fernandez, J.C.; Chervin, C.; Hewezi, T.; Binder, B. Ethylene Receptors Signal via a Noncanonical Pathway to Regulate Abscisic Acid Responses. Plant Physiol. 2017, 176, 910-929. [CrossRef] [PubMed]

33. Wang, H.; Huang, Z.; Chen, Q.; Zhang, Z.; Zhang, H.; Wu, Y.; Huang, D.; Huang, R. Ectopic overexpression of tomato JERF3 in tobacco activates downstream gene expression and enhances salt tolerance. Plant Mol. Biol. 2004, 55, 183-192. [CrossRef] [PubMed]

34. McGrath, K.; Dombrecht, B.; Manners, J.M.; Schenk, P.; Edgar, C.I.; MacLean, D.J.; Scheible, W.-R.; Udvardi, M.K.; Kazan, K. Repressor- and Activator-Type Ethylene Response Factors Functioning in Jasmonate Signaling and Disease Resistance Identified via a Genome-Wide Screen of Arabidopsis Transcription Factor Gene Expression[w]. Plant Physiol. 2005, 139, 949-959. [CrossRef]

35. Zhang, H.; Zhang, J.; Quan, R.; Pan, X.; Wan, L.; Huang, R. EAR motif mutation of rice OsERF3 alters the regulation of ethylene biosynthesis and drought tolerance. Planta 2013, 237, 1443-1451. [CrossRef]

36. Liu, M.; Diretto, G.; Pirrello, J.; Roustan, J.-P.; Li, Z.; Giuliano, G.; Regad, F.; Bouzayen, M. The chimeric repressor version of an Ethylene Response Factor ( ERF ) family member, Sl-ERF.B3, shows contrasting effects on tomato fruit ripening. New Phytol. 2014, 203, 206-218. [CrossRef]

37. Xie, X.-L.; Yin, X.-R.; Chen, K.-S. Roles of APETALA2/Ethylene-Response Factors in Regulation of Fruit Quality. Crit. Rev. Plant Sci. 2016, 35, 120-130. [CrossRef]

38. Zhang, Z.; Zhang, H.; Quan, R.; Wang, X.-C.; Huang, R. Transcriptional Regulation of the Ethylene Response Factor LeERF2 in the Expression of Ethylene Biosynthesis Genes Controls Ethylene Production in Tomato and Tobacco1[W][OA]. Plant Physiol. 2009, 150, 365-377. [CrossRef]

39. Li, T.; Jiang, Z.; Zhang, L.; Tan, D.; Wei, Y.; Yuan, H.; Li, T.; Wang, A. Apple (Malus domestica) MdERF2 negatively affects ethylene biosynthesis during fruit ripening by suppressing MdACS1 transcription. Plant J. 2016, 88, 735-748. [CrossRef] 
40. 4Hao, P.-P.; Wang, G.-M.; Cheng, H.-Y.; Ke, Y.-Q.; Qi, K.-J.; Zhang, S.; Zhang, S. Transcriptome analysis unravels an ethylene response factor involved in regulating fruit ripening in pear. Physiol. Plant 2018, 163, 124-135. [CrossRef]

41. Liu, L.; White, M.J.; Macrae, T.H. Transcription factors and their genes in higher plants functional domains, evolution and regulation. J. Biol. Inorg. Chem. 1999, 262, 247-257. [CrossRef] [PubMed]

42. Ohta, M.; Matsui, K.; Hiratsu, K.; Shinshi, H.; Ohme-Takagi, M. Repression domains of class II ERF transcriptional repressors share an essential motif for active repression. Plant Cell 2001, 13, 1959-1968. [CrossRef] [PubMed]

43. Chen, G.; Hu, Z.; Grierson, D. Differential regulation of tomato ethylene responsive factor LeERF3b, a putative repressor, and the activator Pti4 in ripening mutants and in response to environmental stresses. J. Plant Physiol. 2008, 165, 662-670. [CrossRef] [PubMed]

44. Pauwels, L.; Barbero, G.F.; Geerinck, J.; Tilleman, S.; Grunewald, W.; Perez, A.C.; Chico, J.M.; Bossche, R.V.; Sewell, J.; Gil, E. NINJA connects the co-repressor TOPLESS to jasmonate signalling. Nature 2010, 464, 788-791. [CrossRef]

45. Kagale, S.; Rozwadowski, K. EAR motif-mediated transcriptional repression in plants: An underlying mechanism for epigenetic regulation of gene expression. Epigenetics 2011, 6, 141-146. [CrossRef]

46. Mathooko, F.M.; Tsunashima, Y.; Owino, W.Z.; Kubo, Y.; Inaba, A. Regulation of genes encoding ethylene biosynthetic enzymes in peach (Prunus persica L.) fruit by carbon dioxide and 1-methylcyclopropene. Postharvest. Biol. Techol. 2001, 21, 265-281. [CrossRef]

47. Tatsuki, M.; Haji, T.; Yamaguchi, M. The involvement of 1-aminocyclopropane-1-carboxylic acid synthase isogene, Pp-ACS1, in peach fruit softening. J. Exp. Bot. 2006, 57, 1281-1289. [CrossRef]

48. Prinsi, B.; Negri, A.S.; Fedeli, C.; Morgutti, S.; Negrini, N.; Cocucci, M.; Espen, L. Peach fruit ripening: A proteomic comparative analysis of the mesocarp of two cultivars with different flesh firmness at two ripening stages. Phytochemistry 2011, 72, 1251-1262. [CrossRef]

49. Gong, D.; Cao, S.; Sheng, T.; Shao, J.; Song, C.; Wo, F.; Chen, W.; Yang, Z. Effect of blue light on ethylene biosynthesis, signalling and fruit ripening in postharvest peaches. Sci. Hortic. 2015, 197, 657-664. [CrossRef]

50. Zhang, C.H.; Shangguan, L.F.; Ma, R.J.; Sun, X.; Tao, R.; Guo, L.; Korir, N.K.; Yu, M.L. Genome-wide analysis of the AP2/ERF superfamily in peach (Prunus persica). Genet. Mol. Res. 2012, 11, 4789-4809. [CrossRef]

51. Wang, X.; Ding, Y.; Wang, Y.; Pan, L.; Niu, L.; Lu, Z.; Cui, G.; Zeng, W.; Wang, Z. Genes involved in ethylene signal transduction in peach ( Prunus persica) and their expression profiles during fruit maturation. Sci. Hortic. 2017, 224, 306-316. [CrossRef]

52. Jin, J.; Tian, F.; Yang, D.C.; Meng, Y.Q.; Kong, L.; Luo, J.; Gao, G. PlantTFDB 4.0: Toward a central hub for transcription factors and regulatory interactions in plants. Nucleic Acids Res. 2017, 45, D1040-D1045. [CrossRef] [PubMed]

53. Wang, L.; Zhao, S.; Gu, C.; Zhou, Y.; Zhou, H.; Ma, J.; Cheng, J.; Han, Y. Deep RNA-Seq uncovers the peach transcriptome landscape. Plant Mol. Biol. 2013, 83, 365-377. [CrossRef] [PubMed]

54. Busatto, N.; Rahim, A.; Trainotti, L. Peach ripening transcriptomics unveils new and unexpected targets for the improvement of drupe quality. Plant Genom. Plant Biotechnol. 2013, 165-182. [CrossRef]

55. Lü, P.; Yu, S.; Zhu, N.; Chen, Y.-R.; Zhou, B.; Pan, Y.; Tzeng, D.; Fabi, J.P.; Argyris, J.; Garcia-Mas, J.; et al. Genome encode analyses reveal the basis of convergent evolution of fleshy fruit ripening. Nat. Plants 2018, 4 , 784-791.

56. Martel, C.; Vrebalov, J.; Tafelmeyer, P.; Giovannoni, J.J. The tomato MADS-box transcription factor RIPENING INHIBITOR interacts with promoters involved in numerous ripening processes in a COLORLESS NONRIPENING-dependent manner. Plant Physiol. 2011, 157, 1568-1579. [CrossRef] [PubMed]

57. Yanagisawa, S.; Yoo, S.-D.; Sheen, J. Differential regulation of EIN3 stability by glucose and ethylene signalling in plants. Nature 2003, 425, 521-525. [CrossRef]

58. Potuschak, T.; Lechner, E.; Parmentier, Y.; Yanagisawa, S.; Grava, S.; Koncz, C.; Genschik, P. EIN3-Dependent Regulation of Plant Ethylene Hormone Signaling by Two Arabidopsis F Box Proteins. Cell 2003, 115, 679-689. [CrossRef]

59. Guo, H.; Ecker, J.R. Plant responses to ethylene gas are mediated by SCFEBF1/EBF2-dependent proteolysis of EIN3 transcription factor. Cell 2003, 115, 667-677. [CrossRef] 
60. Liu, M.; Pirrello, J.; Kesari, R.; Mila, I.; Roustan, J.-P.; Li, Z.; Latché, A.; Pech, J.; Bouzayen, M.; Regad, F. A dominant repressor version of the tomato Sl-ERF. B3 gene confers ethylene hypersensitivity via feedback regulation of ethylene signaling and response components. Plant J. 2013, 76, 406-419. [CrossRef]

61. Pirrello, J.; Jaimes-Miranda, F.; Sanchez-Ballesta, M.T.; Tournier, B.; Khalil-Ahmad, Q.; Regad, F.; Latché, A.; Pech, J.; Bouzayen, M. Sl-ERF2, a Tomato Ethylene Response Factor Involved in Ethylene Response and Seed Germination. Plant Cell Physiol. 2006, 47, 1195-1205. [CrossRef] [PubMed]

62. Han, Y.-C.; Kuang, J.-F.; Chen, J.-Y.; Liu, X.; Xiao, Y.-Y.; Fu, C.-C.; Wang, J.-N.; Wu, K.-Q.; Lu, W.-J. Banana Transcription Factor MaERF11 Recruits Histone Deacetylase MaHDA1 and Represses the Expression of MaACO1 and Expansins during Fruit Ripening. Plant Physiol. 2016, 171, 1070-1084. [CrossRef] [PubMed]

63. Howe, E.A.; Sinha, R.; Schlauch, D.; Quackenbush, J. RNA-Seq analysis in MeV. Bioinformatics 2011, 27, 3209-3210. [CrossRef] [PubMed]

64. Tong, Z.; Gao, Z.; Wang, F.; Jun, Z.; Zhang, Z. Selection of reliable reference genes for gene expression studies in peach using real-time PCR. BMC Mol. Biol. 2009, 10, 71. [CrossRef] [PubMed]

65. Hellens, R.; Edwards, E.A.; Leyland, N.; Bean, S.; Mullineaux, P. pGreen: A versatile and flexible binary Ti vector for Agrobacterium-mediated plant transformation. Plant Mol. Biol. 2000, 42, 819-832. [CrossRef]

66. Espley, R.; Hellens, R.P.; Putterill, J.; Stevenson, D.E.; Kutty-Amma, S.; Allan, A.C. Red colouration in apple fruit is due to the activity of the MYB transcription factor, MdMYB10. Plant J. 2007, 49, 414-427. [CrossRef]

(C) 2020 by the authors. Licensee MDPI, Basel, Switzerland. This article is an open access article distributed under the terms and conditions of the Creative Commons Attribution (CC BY) license (http://creativecommons.org/licenses/by/4.0/). 